\title{
INVERSE DEGREE OF AN AFFINE SPACE TRIANGULAR AUTOMORPHISM
}

\author{
SHU KAWAGUCHI
}

(Communicated by Harm Derksen)

\begin{abstract}
For any $\mathbb{Q}$-algebra $R$ and any triangular automorphism $f: R^{N} \rightarrow$ $R^{N}$ with Jacobian one on the affine space, we show that $\operatorname{deg}\left(f^{-1}\right)$ is bounded from above by a constant depending only on $N$ and $\operatorname{deg}(f)$. This is seen as a generalization of a result by Furter on the affine plane. Our proof uses (a version of) Furter's estimate on nilpotency indices and Abhyankar-Gurjar's formal inversion formula. It follows that when the Jacobian of a triangular automorphism $f: R^{N} \rightarrow R^{N}$ is not necessarily equal to one, $\operatorname{deg}\left(f^{-1}\right)$ is bounded from above by a constant depending only on $N, \operatorname{deg}(f)$ and $\operatorname{deg}(1 / \operatorname{Jac}(f))$.
\end{abstract}

\section{INTRODUCTION}

For a $\mathbb{Q}$-algebra $R$, let $R\left[X_{1}, \ldots, X_{N}\right]$ denote the polynomial ring with coefficients in $R$ in $N$ variables. A map $f=\left(f_{1}, \ldots, f_{N}\right): R^{N} \rightarrow R^{N}$ is a polynomial map over $R$ if each $f_{i}$ belongs to $R\left[X_{1}, \ldots, X_{N}\right]$. We denote the Jacobian matrix of $f$ by $J(f)=\left(\frac{\partial f_{i}}{\partial X_{j}}\right)_{1 \leq i, j \leq N} \in \operatorname{Mat}_{N, N}\left(R\left[X_{1}, \ldots, X_{N}\right]\right)$, the Jacobian determinant of $f$ by $\operatorname{Jac}(f)=\operatorname{det} J(f) \in R\left[X_{1}, \ldots, X_{N}\right]$, and the degree of $f$ by $\operatorname{deg}(f)=$ $\max \left\{\operatorname{deg}\left(f_{1}\right), \ldots, \operatorname{deg}\left(f_{N}\right)\right\} \in \mathbb{Z}_{\geq 0}$. A polynomial map $f: R^{N} \rightarrow R^{N}$ over $R$ is an automorphism (i.e. a polynomial automorphism) if it has a polynomial inverse $g: R^{N} \rightarrow R^{N}$ over $R$, i.e., $g \circ f=f \circ g=\operatorname{Id}_{R^{N}}$. If this is the case, $g$ is uniquely determined by $f$ and is denoted by $f^{-1}$.

Let $f: R^{N} \rightarrow R^{N}$ be an automorphism over $R$ with $\operatorname{Jac}(f)=1$. An interesting problem is to bound $\operatorname{deg}\left(f^{-1}\right)$ from above. If $R$ is a reduced $\mathbb{Q}$-algebra, then a formula of Gabber (see [3, I.1] and [4]) says that $\operatorname{deg}\left(f^{-1}\right) \leq \operatorname{deg}(f)^{N-1}$ (here the condition $\operatorname{Jac}(f)=1$ is not necessary). However, if one allows $R$ to be non-reduced, then Bass [2] has shown that the problem of bounding $\operatorname{deg}\left(f^{-1}\right)$ from above by a constant depending only on $N$ and $\operatorname{deg}(f)$ (and independent of non-reduced $\mathbb{Q}$ algebras $R$ ) is, in fact, equivalent to the Jacobian conjecture over $\mathbb{C}$.

In dimension $N=2$, instead of treating all polynomial automorphisms, Furter 8 has considered triangular automorphisms. Recall that $f=\left(f_{1}, \ldots, f_{N}\right): R^{N} \rightarrow R^{N}$ is said to be triangular if $\frac{\partial f_{i}}{\partial X_{j}}=0$ for any $1 \leq i<j \leq N$. The triangular automorphisms constitute an important subgroup of the automorphism group of $R^{N}$. Furter has proved that if $R$ is any $\mathbb{Q}$-algebra and $f: R^{2} \rightarrow R^{2}$ is any triangular automorphism over $R$ with $\operatorname{Jac}(f)=1$, then $\operatorname{deg}\left(f^{-1}\right) \leq 4 \operatorname{deg}(f)^{4}$. (For other

Received by the editors October 18, 2010 and, in revised form, December 13, 2011.

2010 Mathematics Subject Classification. Primary 08A35, 13B25, 14J50, 14R10.

Key words and phrases. Automorphism, degree, inverse, polynomial ring, non-reduced ring.

This work is partially supported by KAKENHI 21740018. 
directions of results related to inverse degrees of affine space automorphisms, see for example [4, 5], 6, Chap. 2.3 and 7.3] and the references in the Notes therein, [7, 9].)

The purpose of this short note is to consider inverse degrees of triangular automorphisms with Jacobian one in arbitrary dimension.

Theorem 1.1. Let $N \geq 1$ and $d \geq 1$. Then there exists a constant $t(N, d)$ which depends only on $N$ and d satisfying the following property: If $R$ is any $\mathbb{Q}$-algebra, and $f=\left(f_{1}, f_{2}, \ldots, f_{N}\right): R^{N} \rightarrow R^{N}$ is any triangular automorphism over $R$ of degree at most $d$ with $\operatorname{Jac}(f)=1$, then $\operatorname{deg}\left(f^{-1}\right) \leq t(N, d)$.

For an explicit form of $t(N, d)$, see the end of the proof of Theorem 1.1. In $N=2$, our proof still gives $\operatorname{deg}\left(f^{-1}\right) \leq 4 \operatorname{deg}(f)^{4}$ but a slightly weaker estimate than Furter [8], where it is shown that $\operatorname{deg}\left(f^{-1}\right) \leq(2 d-1)\left((d-1)^{2}(2 d-3)+1\right) \leq 4 d^{4}$. Our estimate gives $\operatorname{deg}\left(f^{-1}\right) \leq(2 d+2)\left((d-1)^{2}(2 d-3)+1\right)-d-2 \leq 4 d^{4}$ if $d \geq 2$ (see Remark 2.7).

Key ingredients of our proof are (a version in $N$ variables of) Furter's estimate on nilpotency indices (Proposition 2.4) and Abhyankar-Gurjar's formal inversion formula (Theorem 2.2 and Corollary 2.3) .

Suppose $f: R^{N} \rightarrow R^{N}$ is an automorphism over $R$. Then it follows from $1=\operatorname{Jac}\left(f^{-1} \circ f\right)=\operatorname{Jac}\left(f^{-1}\right)(f) \cdot \operatorname{Jac}(f)$ that $\operatorname{Jac}(f)$ is a unit in $R\left[X_{1}, \ldots, X_{N}\right]$. Thus $1 / \operatorname{Jac}(f) \in R\left[X_{1}, \ldots, X_{N}\right]$, and we can consider $\operatorname{deg}(1 / \operatorname{Jac}(f))$. Theorem 1.1 tells us that in the case $\operatorname{Jac}(f)$ is not necessarily equal to one, $\operatorname{deg}\left(f^{-1}\right)$ is then bounded from above by a constant depending only on $N, \operatorname{deg}(f)$ and $\operatorname{deg}(1 / \operatorname{Jac}(f))$.

Corollary 1.2. Let $N \geq 1, d \geq 1$ and $e \geq 0$. Then there exists a constant $t(N, d, e)$ which depends only on $N, d$ and $e$ satisfying the following property: If $R$ is any $\mathbb{Q}$-algebra, and $f=\left(f_{1}, f_{2}, \ldots, f_{N}\right): R^{N} \rightarrow R^{N}$ is any triangular automorphism over $R$ of degree at most $d$ with $\operatorname{deg}(1 / \operatorname{Jac}(f)) \leq e$, then $\operatorname{deg}\left(f^{-1}\right) \leq t(N, d, e)$.

Finally, we remark that some condition on $\operatorname{deg}(1 / \operatorname{Jac}(f))(\operatorname{such}$ as $\operatorname{Jac}(f)=1$ in Theorem 1.1. or $\operatorname{deg}(1 / \operatorname{Jac}(f)) \leq e$ in general $)$ is necessary. Indeed, for any $d \geq 2, d^{\prime} \geq 2$, by [8, Lemma 1], if one drops the condition $\operatorname{Jac}(f)=1$, then there exists a triangular automorphism $f: R^{N} \rightarrow R^{N}$ over $R$ of degree $d$ such that $\operatorname{deg}\left(f^{-1}\right)=d^{\prime}$.

\section{Proof}

Let $R$ be a $\mathbb{Q}$-algebra. Let $f=\left(f_{1}\left(X_{1}\right), f_{2}\left(X_{1}, X_{2}\right), \ldots, f_{N}\left(X_{1}, X_{2}, \ldots, X_{N}\right)\right)$ : $R^{N} \rightarrow R^{N}$ be a triangular automorphism, where $f_{i}=f_{i}\left(X_{1}, \ldots, X_{i}\right) \in R\left[X_{1}, \ldots\right.$, $\left.X_{i}\right]$. We assume that $\operatorname{Jac}(f)=1$. Let $g=\left(g_{1}, \ldots, g_{N}\right): R^{N} \rightarrow R^{N}$ denote the inverse of $f$.

Lemma 2.1. The automorphism $g$ is triangular with $\operatorname{Jac}(g)=1$.

Proof. Since $1=\operatorname{Jac}(f \circ g)=\operatorname{Jac}(f)(g) \cdot \operatorname{Jac}(g)$, we have $\operatorname{Jac}(g)=1$. We will show that $g$ is a triangular automorphism.

Since $\operatorname{Jac}(f)=1$ and $f$ is triangular, we have

$$
\frac{\partial f_{1}}{\partial X_{1}}\left(X_{1}\right) \cdot \frac{\partial f_{2}}{\partial X_{2}}\left(X_{1}, X_{2}\right) \cdots \frac{\partial f_{N}}{\partial X_{N}}\left(X_{1}, X_{2}, \ldots, X_{N}\right)=1
$$

It follows that for each $i, \frac{\partial f_{i}}{\partial X_{i}}\left(X_{1}, \ldots, X_{i}\right)$ is a unit in $R\left[X_{1}, \ldots, X_{i}\right]$. 
We write $g_{i}=g_{i}\left(Y_{1}, \ldots, Y_{N}\right)$ for $i=1, \ldots, N$. Since $g \circ f=\operatorname{Id}_{R^{N}}$, we have

$$
g_{i}\left(f_{1}\left(X_{1}\right), \ldots, f_{N}\left(X_{1}, \ldots, X_{N}\right)\right)=X_{i} .
$$

Taking $\frac{\partial}{\partial X_{N}}$ in (2.1), we obtain $\frac{\partial g_{i}}{\partial Y_{N}}\left(f_{1}, \ldots, f_{N}\right) \cdot \frac{\partial f_{N}}{\partial X_{N}}=0$. Since $\frac{\partial f_{N}}{\partial X_{N}}$ is a unit and $f$ is an automorphism of $R\left[X_{1}, \ldots, X_{N}\right]$, we have $\frac{\partial g_{i}}{\partial Y_{N}}\left(Y_{1}, \ldots, Y_{N}\right)=0$. Next, taking $\frac{\partial}{\partial X_{N-1}}$ in (2.1), we obtain

$$
\begin{aligned}
0 & =\frac{\partial g_{i}}{\partial Y_{N-1}}\left(f_{1}, \ldots, f_{N}\right) \cdot \frac{\partial f_{N-1}}{\partial X_{N-1}}+\frac{\partial g_{i}}{\partial Y_{N}}\left(f_{1}, \ldots, f_{N}\right) \cdot \frac{\partial f_{N}}{\partial X_{N-1}} \\
& =\frac{\partial g_{i}}{\partial Y_{N-1}}\left(f_{1}, \ldots, f_{N}\right) \cdot \frac{\partial f_{N-1}}{\partial X_{N-1}} .
\end{aligned}
$$

Since $\frac{\partial f_{N-1}}{\partial X_{N-1}}$ is a unit, we similarly have $\frac{\partial g_{i}}{\partial Y_{N-1}}\left(Y_{1}, \ldots, Y_{N}\right)=0$. We can proceed with the same procedure for any $j \geq i+1$, and we see that $\frac{\partial g_{i}}{\partial Y_{j}}\left(Y_{1}, \ldots, Y_{N}\right)=0$ for any $N \geq j>i \geq 1$. Hence $g$ is triangular.

We are going to give an upper bound for $\operatorname{deg}(g)$. First we recall AbhyankarGurjar's formal inversion formula (see [1] and [3, III.2]).

Theorem 2.2 (Abhyankar-Gurjar's formal inversion formula). Let $f: R^{N} \rightarrow R^{N}$ be a polynomial map over $R$ with $f(0)=0$ and $J(f)(0)=I_{N}$. Put $f=\mathrm{Id}-h$, where $h=\left(h_{1}, \ldots, h_{N}\right)$. Let $g=\left(g_{1}, \ldots, g_{N}\right)$ be the formal power series inverse of $f$. Then as an equality in $R\left[\left[X_{1}, \ldots, X_{N}\right]\right]$, we have

$$
g_{i}=\sum_{p=\left(p_{1}, \ldots, p_{N}\right) \in \mathbb{Z}_{\geq 0}^{N}} \frac{1}{p_{1} ! \cdots p_{N} !}\left(\frac{\partial}{\partial X_{1}}\right)^{p_{1}} \cdots\left(\frac{\partial}{\partial X_{N}}\right)^{p_{N}}\left(X_{i} \cdot \operatorname{Jac}(f) \cdot h_{1}^{p_{1}} \cdots h_{N}^{p_{N}}\right) .
$$

As a corollary, if $X_{i}-f_{i}$ is nilpotent for each $i$, we have the following estimate on the degree of $g_{i}$ (as a polynomial in $R\left[X_{1}, \ldots, X_{N}\right]$ ). This actually gives a generalization of [8, Theorem 1] of $N=1$.

Corollary 2.3. Let $f: R^{N} \rightarrow R^{N}$ be a polynomial map over $R$ of degree at most $d$ with $f(0)=0$ and $J(f)(0)=I_{N}$. Let $g$ be the inverse of $f$. We assume further that there exist $\lambda_{1}, \ldots, \lambda_{N} \geq 1$ with $\left(X_{1}-f_{1}\right)^{\lambda_{1}}=\cdots=\left(X_{N}-f_{N}\right)^{\lambda_{N}}=0$. Then

$$
\operatorname{deg}(g) \leq(d-1) \sum_{k=1}^{N}\left(\lambda_{k}-1\right)+1
$$

Proof. We set $h_{i}=X_{i}-f_{i}$. By Theorem 2.2, we get

$$
g_{i}=\sum_{p=\left(p_{1}, \ldots, p_{n}\right) \in \mathbb{Z}_{\geq 0}^{N}} \frac{1}{p_{1} ! \cdots p_{N} !}\left(\frac{\partial}{\partial X_{1}}\right)^{p_{1}} \cdots\left(\frac{\partial}{\partial X_{N}}\right)^{p_{N}}\left(X_{i} \cdot h_{1}^{p_{1}} \cdots h_{N}^{p_{N}}\right) .
$$

The degree of each term of the right-hand side is at most $\left(\sum_{k=1}^{N} p_{k}\left(\operatorname{deg}\left(h_{k}\right)-1\right)\right)+1$, and it is zero if $p_{k} \geq \lambda_{k}$ for some $k$. Hence the right-hand side is a finite sum, and the above equality holds in $R\left[X_{1}, \ldots, X_{N}\right]$. By degree counting, we get the assertion.

We also recall Furter's estimate on nilpotent indices. For later use, we give a version in $N$ variables. 
Proposition 2.4. Let $N \geq 1, d \geq 1$ and $e \geq 1$. Let

$$
P\left(X_{1}, \ldots, X_{N}\right) \in\left(R\left[X_{1}, \ldots, X_{N}\right]\right)^{\times}
$$

such that $\operatorname{deg}(P) \leq d$ and $\operatorname{deg}\left(P^{-1}\right) \leq e$. We write $P=p_{0}+p_{1} X_{N}+\cdots+p_{d} X_{N}^{d}$, where $p_{i}=p_{i}\left(X_{1}, \ldots, X_{N-1}\right) \in R\left[X_{1}, \ldots, X_{N-1}\right]$. Then

$$
\left(p_{0}-p_{0}(0), p_{1}, \ldots, p_{d}\right)^{N \min \{d, e\}(d+e-1)+1}=0
$$

as an ideal in $R\left[X_{1}, \ldots, X_{N-1}\right]$.

Proof. The case $N=1$ is due to Furter [8, Prop. 1]. For the reader's convenience, we recall the proof of [8. Prop. 1], following the referee's suggestion. Since $P\left(X_{1}\right)=$ $p_{0}+p_{1} X_{1}+\cdots+p_{d} X_{1}^{d}$ is a unit of $R\left[X_{1}\right], p_{0}$ is a unit of $R$. Replacing $P$ by $p_{0}^{-1} P$, we may assume that $p_{0}=1$ to show the assertion. We set the monic polynomial $Q\left(X_{1}\right):=X_{1}^{d} P\left(1 / X_{1}\right)=X_{1}^{d}+p_{1} X_{1}^{d-1}+\cdots+p_{d} \in R\left[X_{1}\right]$ and set $R_{1}:=R[T] /(Q(T))$, where $T$ is indeterminate. Then $R_{1}$ is a $\mathbb{Q}$-algebra containing $R$. We denote by $\varepsilon_{1}$ the image of $T$ in $R_{1}$. Since $Q\left(\varepsilon_{1}\right)=0$ in $R_{1}$, there exists a monic polynomial $Q_{1}\left(X_{1}\right) \in R_{1}\left[X_{1}\right]$ with $\operatorname{deg}\left(Q_{1}\right)=\operatorname{deg}(Q)-1$ such that $Q\left(X_{1}\right)=$ $\left(X_{1}-\varepsilon_{1}\right) Q_{1}\left(X_{1}\right)$ in $R_{1}\left[X_{1}\right]$. By induction on $\operatorname{deg}(Q)$, we see that there exist a $\mathbb{Q}$-algebra $R^{\prime}$ containing $R$ and $\varepsilon_{1}, \ldots, \varepsilon_{d} \in R^{\prime}$ such that $Q\left(X_{1}\right)=\prod_{i=1}^{d}\left(X_{1}-\varepsilon_{i}\right)$ in $R^{\prime}\left[X_{1}\right]$. Then $P\left(X_{1}\right)=X_{1}^{d} Q\left(1 / X_{1}\right)=\prod_{i=1}^{d}\left(1-\varepsilon_{i} X_{1}\right)$ in $R^{\prime}\left[X_{1}\right]$. For each $j$, we have

$$
\left(1-\varepsilon_{j} X_{1}\right)^{-1}=\left(P^{-1}\right)\left(X_{1}\right)\left(\prod_{1 \leq i \leq d, i \neq j}\left(1-\varepsilon_{i} X_{1}\right)\right) .
$$

Since the right-hand side is a polynomial of degree at most $d+e-1$, we have $\varepsilon_{j}^{d+e}=0$ for all $j=1, \ldots, d$. Let $\mathcal{E}$ be the ideal of $R^{\prime}$ generated by $\varepsilon_{1}, \ldots, \varepsilon_{d}$. Then $\mathcal{E}^{d(d+e-1)+1}=0$. Since $\left(p_{1}, \ldots, p_{d}\right) R^{\prime} \subseteq \mathcal{E}$, we have $\left(p_{1}, \ldots, p_{d}\right)^{d(d+e-1)+1}=0$ in $R$. We write $P^{-1}\left(X_{1}\right)=1+q_{1} X_{1}+\cdots+q_{e} X_{1}^{e}$. Interchanging $P$ and $P^{-1}$, we have $\left(q_{1}, \ldots, q_{e}\right)^{e(d+e-1)+1}=0$ in $R$. Since $\left(p_{1}, \ldots, p_{d}\right)=\left(q_{1}, \ldots, q_{e}\right)$, we have $\left(p_{1}, \ldots, p_{d}\right)^{\min \{d, e\}(d+e-1)+1}=0$, which gives the assertion in $N=1$.

Suppose now that $N \geq 2$. Since $\operatorname{deg}_{X_{N}}(P) \leq d$ and $\operatorname{deg}_{X_{N}}\left(P^{-1}\right) \leq e$, the case $N=1$ applied to $R\left[X_{1}, \ldots, X_{N}\right]=\left(R\left[X_{1}, \ldots, X_{N-1}\right]\right)\left[X_{N}\right]$, regarded as the one variable polynomial ring over $R\left[X_{1}, \ldots, X_{N-1}\right]$ with indeterminate $X_{N}$, gives

$$
\left(p_{1}, \ldots, p_{d}\right)^{\min \{d, e\}(d+e-1)+1}=0 .
$$

Since $p_{0}\left(X_{1}, \ldots, X_{N-1}\right)=P\left(X_{1}, \ldots, X_{N-1}, 0\right)$, we have $p_{0}\left(X_{1}, \ldots, X_{N-1}\right) \in$ $\left(R\left[X_{1}, \ldots, X_{N-1}\right]\right)^{\times}$and $\operatorname{deg}\left(p_{0}\right) \leq d$ and $\operatorname{deg}\left(p_{0}^{-1}\right) \leq e$. By induction on $N$, we get $\left(p_{0}-p_{0}(0)\right)^{(N-1) \min \{d, e\}(d+e-1)+1}=0$. Since $N \min \{d, e\}(d+e-1)+1=$ $(\min \{d, e\}(d+e-1)+1)+((N-1) \min \{d, e\}(d+e-1)+1)-1$, we have

$$
\left(p_{0}-p_{0}(0), p_{1}, \ldots, p_{d}\right)^{N \min \{d, e\}(d+e-1)+1}=0 .
$$

This gives the assertion.

Proposition 2.5. Let $N \geq 2$. Let $f=\left(f_{1}, f_{2}, \ldots, f_{N}\right): R^{N} \rightarrow R^{N}$ be a triangular automorphism over $R$ of degree at most d with $f(0)=0, J(f)(0)=I_{N}$ and $\operatorname{Jac}(f)=$ 1. Let $g$ be the inverse of $f$. We assume further that $f_{i}\left(X_{1}, \ldots, X_{i-1}, 0\right)=0$ for all $1 \leq i \leq N$. Then

$$
\operatorname{deg}(g) \leq \frac{(N+2)(N-1)}{2}(d-1)^{N}\left((d-1)^{N-1}+d-2\right)+1 .
$$


Proof. As in the proof of Lemma 2.1, we have

$$
\frac{\partial f_{1}}{\partial X_{1}}\left(X_{1}\right) \cdot \frac{\partial f_{2}}{\partial X_{2}}\left(X_{1}, X_{2}\right) \cdots \frac{\partial f_{N}}{\partial X_{N}}\left(X_{1}, X_{2}, \ldots, X_{N}\right)=1
$$

Putting $X_{k+1}=\cdots=X_{N}=0$, we get

$$
\frac{\partial f_{1}}{\partial X_{1}}\left(X_{1}\right) \cdots \frac{\partial f_{k}}{\partial X_{k}}\left(X_{1}, \ldots, X_{k}\right) \cdots \frac{\partial f_{N}}{\partial X_{N}}\left(X_{1}, \ldots, X_{k}, 0, \ldots, 0\right)=1 .
$$

It follows that $\operatorname{deg}\left(\frac{\partial f_{k}}{\partial X_{k}}\left(X_{1}, \ldots, X_{k}\right)\right) \leq(d-1)$ and $\operatorname{deg}\left(\frac{\partial f_{k}}{\partial X_{k}}\left(X_{1}, \ldots, X_{k}\right)^{-1}\right) \leq$ $(d-1)^{N-1}$.

Let $k \leq N-1$. We write

$$
\frac{\partial f_{k}}{\partial X_{k}}\left(X_{1}, \ldots, X_{k}\right)=p_{0}+p_{1} X_{k}+\cdots+p_{d-1} X_{k}^{d-1},
$$

where $p_{k}=p_{k}\left(X_{1}, \ldots, X_{k-1}\right) \in R\left[X_{1}, \ldots, X_{k-1}\right]$. By Proposition 2.4, we have

$$
\left(p_{0}-p_{0}(0), p_{1}, \ldots, p_{d-1}\right)^{k(d-1)^{N-1}\left((d-1)^{N-1}+d-2\right)+1}=0 .
$$

Since $J(f)(0)=I_{N}$, we get $p_{0}(0)=1$. Further, since $f_{k}\left(X_{1}, \ldots, X_{k-1}, 0\right)=0$, we have

$$
f_{k}\left(X_{1}, \ldots, X_{k}\right)=X_{k}+\left(p_{0}-p_{0}(0)\right) X_{k}+\frac{1}{2} p_{1} X_{k}^{2}+\cdots+\frac{1}{d} p_{d-1} X_{k}^{d} .
$$

It follows that $\left(X_{k}-f_{k}\right)^{k(d-1)^{N-1}\left((d-1)^{N-1}+d-2\right)+1}=0$.

Let $k=N$. Equation (2.2) tells us that $\frac{\partial f_{N}}{\partial X_{N}}$ belongs to $R\left[X_{1}, \ldots, X_{N-1}\right]$. We write

$$
\frac{\partial f_{N}}{\partial X_{N}}=q_{0}+q_{1} X_{N-1}+\cdots+q_{d-1} X_{N-1}^{d-1},
$$

where $q_{k}=q_{k}\left(X_{1}, \ldots, X_{N-2}\right) \in R\left[X_{1}, \ldots, X_{N-2}\right]$. By Proposition 2.4, we have

$$
\left(q_{0}-q_{0}(0), q_{1}, \ldots, q_{d-1}\right)^{(N-1)(d-1)^{N-1}\left((d-1)^{N-1}+d-2\right)+1}=0 .
$$

Since $J(f)(0)=I_{N}$, we get $q_{0}(0)=1$. Further, since $f_{N}\left(X_{1}, \ldots, X_{N-1}, 0\right)=0$, we have $f_{N}\left(X_{1}, \ldots, X_{N}\right)=X_{N}+\left(\frac{\partial f_{N}}{\partial X_{N}}-q_{0}(0)\right) X_{N}$. It follows that

$$
\left(X_{N}-f_{N}\right)^{(N-1)(d-1)^{N-1}\left((d-1)^{N-1}+d-2\right)+1}=0 .
$$

By Corollary 2.3, we get

$$
\begin{aligned}
\operatorname{deg}(g) & \leq\left(\sum_{k=1}^{N-1} k+(N-1)\right)(d-1)^{N}\left((d-1)^{N-1}+d-2\right)+1 \\
& =\frac{(N+2)(N-1)}{2}(d-1)^{N}\left((d-1)^{N-1}+d-2\right)+1 .
\end{aligned}
$$

This completes the proof.

Lemma 2.6. For $1 \leq i \leq N-1$, let $s_{i}\left(X_{1}, \ldots, X_{i}\right) \in R\left[X_{1}, \ldots, X_{i}\right]$ be polynomials of degree at most $d$. We consider a triangular automorphism

$$
t=\left(X_{1}, X_{2}+s_{1}\left(X_{1}\right), \ldots, X_{n}+s_{n-1}\left(X_{1}, \ldots, X_{n-1}\right)\right): R^{N} \rightarrow R^{N} .
$$

Then $\operatorname{deg}\left(t^{-1}\right) \leq d^{N-1}$. 
Proof. Let $u=\left(u_{1}, \ldots, u_{N}\right)$ be the inverse of $t$. We show that $\operatorname{deg}\left(u_{i}\right) \leq d^{i-1}$. Since $u_{1}\left(X_{1}\right)=X_{1}$, we have $\operatorname{deg}\left(u_{1}\right) \leq 1=d^{1-1}$. Since $u_{i}\left(X_{1}, \ldots, X_{i}\right)=X_{i}-$ $s_{i}\left(u_{1}, \ldots, u_{i-1}\right)$, we have by induction $\operatorname{deg}\left(u_{i}\right) \leq d \cdot d^{i-2}=d^{i-1}$. This gives the assertion.

Proof of Theorem 1.1. Since the case $N=1$ is obvious, we consider the case $N \geq 2$.

First note that we may assume $f(0)=0$ and $J(f)(0)=I_{N}$. Indeed, we put $J(f)(0)=\left(a_{i j}\right)$. Since $J(f)(0)$ is a triangular matrix and $\operatorname{Jac}(f)=1$, we get $\prod_{i} a_{i i}=1$. Thus each $a_{i i}$ is a unit of $R$. It follows that there exists a linear triangular automorphism $L: R^{N} \rightarrow R^{N}$ such that $\tilde{f}:=L(f-f(0))$ is a triangular automorphism with $\tilde{f}(0)=0, J(\tilde{f})(0)=I_{N}$ and $\operatorname{Jac}(\widetilde{f})=1$. Since $\operatorname{deg}\left(\tilde{f}^{-1}\right)=$ $\operatorname{deg}\left(f^{-1}\right)$, it suffices to prove Theorem 1.1 for $\tilde{f}$. Replacing $\tilde{f}$ by $f$, this shows that we may assume $f(0)=0$ and $J(f)(0)=I_{N}$. In this case, the inverse $g$ of $f$ also satisfies $g(0)=0$ and $J(g)(0)=I_{N}$.

We set $\varphi_{1}\left(X_{1}\right)=f_{1}\left(X_{1}\right)$ and, for $i \geq 2$,

$$
\varphi_{i}\left(X_{1}, \ldots, X_{i}\right)=f_{i}\left(X_{1}, \ldots, X_{i}\right)-f_{i}\left(X_{1}, \ldots, X_{i-1}, 0\right),
$$

and consider the polynomial map $\varphi=\left(\varphi_{1}, \ldots, \varphi_{N}\right): R^{N} \rightarrow R^{N}$. Then $\varphi$ is a triangular map of degree at most $d$ with $\varphi(0)=0, J(\varphi)(0)=I_{N}$ and $\operatorname{Jac}(\varphi)=1$. We note that $\varphi$ is an automorphism. Indeed, suppose that $Y_{1}=\varphi_{1}\left(X_{1}\right), \ldots, Y_{N}=$ $\varphi\left(X_{1}, \ldots, X_{N}\right)$. Since $f$ is a triangular automorphism, $Y_{1}$ is written as a polynomial in $X_{1}$. Since $Y_{i}+f_{i}\left(X_{1}, \ldots, X_{i-1}, 0\right)=f_{i}\left(X_{1}, \ldots, X_{i-1}, X_{i}\right)$, we have $X_{i}=g_{i}\left(Y_{1}, \ldots, Y_{i}+f_{i}\left(X_{1}, \ldots, X_{i-1}, 0\right)\right)$. Then, inductively, $X_{i}$ is written as a polynomial in $Y_{1}, \ldots, Y_{i}$, which shows that $\varphi$ is an automorphism.

Let $\psi=\left(\psi_{1}, \ldots, \psi_{N}\right)$ denote the inverse of $\varphi$. We set $t_{1}\left(X_{1}\right)=X_{1}$ and, for $i \geq 2$,

$$
t_{i}\left(X_{1}, \ldots, X_{i}\right)=X_{i}+f_{i}\left(\psi_{1}\left(X_{1}\right), \ldots, \psi_{i-1}\left(X_{1}, \ldots, X_{i-1}\right), 0\right),
$$

and consider the polynomial map $t=\left(t_{1}, \ldots t_{N}\right): R^{N} \rightarrow R^{N}$. Then $t$ is a triangular automorphism, and we have

$$
\begin{aligned}
t_{i}\left(\varphi_{1}, \ldots, \varphi_{i}\right) & =\varphi_{i}+f_{i}\left(\psi_{1}\left(\varphi_{1}\right), \ldots, \psi_{i-1}\left(\varphi_{1}, \ldots, \varphi_{i-1}\right), 0\right) \\
& =\varphi_{i}+f_{i}\left(X_{1}, \ldots, X_{i-1}, 0\right)=f_{i}\left(X_{1}, \ldots, X_{i}\right) .
\end{aligned}
$$

Thus $f=t \circ \varphi$.

The inverse $f$ is given by $\psi \circ t^{-1}$. Since $\varphi$ satisfies the assumption of Proposition 2.5, we have

$$
\operatorname{deg}(\psi) \leq \frac{(N+2)(N-1)}{2}(d-1)^{N}\left((d-1)^{N-1}+d-2\right)+1 .
$$

Thus $\operatorname{deg}(t) \leq d\left(\frac{(N+2)(N-1)}{2}(d-1)^{N}\left((d-1)^{N-1}+d-2\right)+1\right)$. Then Lemma 2.6 tells us that $\operatorname{deg}\left(t^{-1}\right) \leq d^{N-1}\left(\frac{(N+2)(N-1)}{2}(d-1)^{N}\left((d-1)^{N-1}+d-2\right)+1\right)^{N-1}$.

Finally we have

$$
\begin{aligned}
\operatorname{deg}\left(f^{-1}\right) & \leq \operatorname{deg}(\psi) \cdot \operatorname{deg}\left(t^{-1}\right) \\
& \leq d^{N-1}\left(\frac{(N+2)(N-1)}{2}(d-1)^{N}\left((d-1)^{N-1}+d-2\right)+1\right)^{N} .
\end{aligned}
$$

This completes the proof of Theorem 1.1 
Remark 2.7. Let $d \geq 2$. In $N=2, \operatorname{Jac}(\psi)=1$ gives $\frac{\partial \psi_{1}}{\partial X_{1}} \cdot \frac{\partial \psi_{2}}{\partial X_{2}}=1$. Thus $\frac{\partial \psi_{2}}{\partial X_{2}}$ does not depend on $X_{2}$, so that $\operatorname{deg}_{X_{2}}\left(\psi_{2}\right)=1$. Then we find that

$$
\begin{aligned}
\operatorname{deg}\left(g_{1}\right) & \leq \operatorname{deg}\left(\psi_{1}\left(X_{1}\right)\right) \leq 2(d-1)^{2}(2 d-3), \\
\operatorname{deg}\left(g_{2}\right) & =\operatorname{deg}\left(\psi_{2}\left(X_{1}, X_{2}-f_{2}\left(\psi_{1}\left(X_{1}\right), 0\right)\right)\right) \\
& \leq \operatorname{deg}\left(X_{2}-f_{2}\left(\psi_{1}\left(X_{1}\right), 0\right)\right)+\left(\operatorname{deg}\left(\psi_{2}\right)-1\right) \\
& =d\left(2(d-1)^{2}(2 d-3)+1\right)+2(d-1)^{2}(2 d-3) \\
& =(2 d+2)\left((d-1)^{2}(2 d-3)+1\right)-d-2 .
\end{aligned}
$$

It follows that we still have $\operatorname{deg}\left(f^{-1}\right) \leq(2 d+2)\left((d-1)^{2}(2 d-3)+1\right)-d-2 \leq 4 d^{4}$.

Proof of Corollary [1.2, Let $f=\left(f_{1}, f_{2}, \ldots, f_{N}\right): R^{N} \rightarrow R^{N}$ be a triangular automorphism over $R$ of degree at most $d$ with $\operatorname{deg}(1 / \operatorname{Jac}(f)) \leq e$. Let $g=\left(g_{1}, \ldots, g_{N}\right)$ denote the inverse of $f$.

From $f$, we construct a triangular automorphism $\tilde{f}=\left(\widetilde{f}_{1}, \ldots, \widetilde{f}_{N}, \widetilde{f}_{N+1}\right): R^{N+1}$ $\rightarrow R^{N+1}$ as follows. For $i=1, \ldots, N$, we set $\widetilde{f}_{i}=f_{i}$. For $i=N+1$, we set

$$
\tilde{f}_{N+1}=X_{N+1} \cdot(1 / \operatorname{Jac}(f)) .
$$

Then $\tilde{f}$ is a triangular map. If we set $\widetilde{g}_{i}=g_{i}$ for $i=1, \ldots, N$ and $\widetilde{g}_{N+1}=$ $X_{N+1} \cdot \operatorname{Jac}(f)\left(g_{1}, \ldots, g_{N}\right)$, then $\widetilde{f}^{-1}$ is given by $\left(\widetilde{g}_{1}, \ldots, \widetilde{g}_{N}, \widetilde{g}_{N+1}\right)$. Thus $\tilde{f}$ is a triangular automorphism. Further, we have $\operatorname{Jac}(\widetilde{f})=\operatorname{Jac}(f) \cdot(1 / \operatorname{Jac}(f))=1$. Since $\tilde{f}: R^{N+1} \rightarrow R^{N+1}$ is a triangular automorphism over $R$ of degree at most $\max \{d, e+1\}$ with $\operatorname{Jac}(\widetilde{f})=1$, Theorem 1.1 shows that

$$
\operatorname{deg}\left(\widetilde{f}^{-1}\right) \leq t(N+1, \max \{d, e+1\}) .
$$

In particular,

$$
\begin{aligned}
\operatorname{deg}\left(f^{-1}\right) & =\max \left\{g_{1}, \ldots, g_{N}\right\}=\max \left\{\widetilde{g}_{1}, \ldots, \widetilde{g}_{N}\right\} \\
& \leq \max \left\{\widetilde{g}_{1}, \ldots, \widetilde{g}_{N}, \widetilde{g}_{N+1}\right\}=\operatorname{deg}\left(\widetilde{f}^{-1}\right) \leq t(N+1, \max \{d, e+1\}) .
\end{aligned}
$$

If we put $t(N, d, e):=t(N+1, \max \{d, e+1\})$, then we obtain the assertion.

\section{ACKNOWLEDGMENT}

The author thanks the referee for carefully reading the manuscript and giving helpful comments.

\section{REFERENCES}

[1] S. S. Abhyankar, Lectures in algebraic geometry. Notes by Chris Christensen, Purdue University, 1974.

[2] H. Bass, The Jacobian conjecture and inverse degrees, Arithmetic and geometry, Vol. II, 65-75, Progr. Math., 36, Birkhäuser, Boston, Mass., 1983. MR717606 (84k:13007)

[3] H. Bass, E.H. Connel, and D. Wright, The Jacobian conjecture: reduction of degree and formal expansion of the inverse, Bull. Amer. Math. Soc. (N.S.) 7 (1982), no. 2, 287-330. MR663785 (83k:14028)

[4] C. C.-A. Cheng, S. S.-S. Wang, J.-T. Yu, Degree bounds for inverses of polynomial automorphisms, Proc. Amer. Math. Soc. 120 (1994), no. 3, 705-707. MR.1195715 (94e:14016)

[5] H. Derksen, Inverse degrees and the Jacobian conjecture, Comm. Algebra 22 (1994), no. 12, 4793-4794. MR:1285708 (95b:14011) 
[6] A. van den Essen, Polynomial automorphisms and the Jacobian conjecture, Progress in Mathematics, 190. Birkhäuser Verlag, Basel, 2000. MR1790619 (2001j:14082)

[7] M. Fouenié, J.-Ph. Furter and D. Pinchon, Computation of the maximal degree of the inverse of a cubic automorphism of the affine plane with Jacobian 1 via Gröbner bases, J. Symbolic Comput. 26 (1998), no. 3, 381-386. MR.1633884 (2000b:14085)

[8] J.-Ph. Furter, On the degree of the inverse of an automorphism of the affine plane, J. Pure Appl. Algebra 130 (1998), no. 3, 277-292. MR1633771 (99g:14015)

[9] S. Maubach, The automorphism group of $\mathbb{C}[T] /\left(T^{m}\right)\left[X_{1}, \ldots, X_{n}\right]$, Comm. Algebra 30 (2002), no. 2, 619-629. MR1883016(2002k:14092)

Department of Mathematics, Graduate School of Science, Osaka University, ToyONAKA, OSAKA 560-0043, JAPAN

E-mail address: kawaguch@math.sci.osaka-u.ac.jp

Current address: Department of Mathematics, Graduate School of Science, Kyoto University, Kyoto 606-8502, Japan

E-mail address: kawaguch@math.kyoto-u.ac.jp 